Ann. Génét. Sél. anim., x969, 1 (I), 7I-77.

\title{
NOTE SUR L'UTILISATION DES RÉSULTATS DES ÉPREUVES DE DESCENDANCE PAR LES SÉLECTIONNEURS DE PORC EN FRANCE DE 1956 A 1965
}

\author{
Zofia J. CZAJEWSKA et L. OLLIVIER \\ Station de Génétique quantitative el appliquée, \\ Centre national de Recherches zootechniques, 78 -Jouy-en-Josas \\ Institut national de la Recherche agronomique
}

\section{SOMMAIRE}

Une méthode est proposée qui permet de mesurer objectivement l'intensité de la sélection pratiquée en fonction des résultats des épreuves de descendance. Il apparait que, de 1956 à 1965 , les sélectionneurs de porcs en France n'ont, en général, guère tenu compte de ces résultats dans le choix de leurs reproducteurs.

Si la sélection a été faible, on constate cependant qu'elle a été plus forte pour les critères de carcasse que pour les critères d'engraissement, plus forte dans le choix des mâles que dans celui des femelles et plus forte dans la race Large White que dans la race Landrace.

\section{INTRODUCTION}

L'épreuve de la descendance est un outil de sélection largement utilisé chez le porc. Suivant l'exemple du Danemark, la plupart des pays européens l'ont adoptée. En France, l'épreuve de la descendance a été mise à la disposition des sélectionneurs en 1953 .

Cette technique suppose des investissements importants et, en conséquence, il est souhaitable de connaître l'usage qu'en font ceux à qui elle est destinée. Nous nous proposons ici de mesurer objectivement l'intensité de la sélection qui a été pratiquée en fonction des résultats des épreuves de descendance, en France, de 1956 à r 965 . 


\section{METTHODES}

\section{a) Principe de l'épreuve de descendance et classement des verrats}

La mise à l'épreuve d'un verrat sur sa descendance requiert l'envoi par l'éleveur de 3 groupes de 3 descendants, chaque groupe étant constitué d'un mâle castré et de 2 femelles issus d'une même portée. La comparaison des verrats entre eux porte sur 4 critères, le gain moyen quotidien de 25 à 100 kilogrammes, l'indice de transformation entre 25 et 100 kilogrammes (c'est-à-dire le nombre de $\mathrm{kg}$ d'aliment consommé par $\mathrm{kg}$ de gain de poids net), le pourcentage de morceaux nobles (jambon et longe) et de morceaux gras (bardière et panne) dans la carcasse. Pour chaque critère, la moyenne du verrat est comparée à une moyenne annuelle de référence (établie pour chaque race et dans chaque porcherie). Ces moyennes sont calculées comme suit : 1/3 (moyenne des mâles) $+2 / 3$ (moyenne des femelles) pour éliminer les variations dues au sexe. Le verrat reçoit une note pour chaque critère selon le principe suivant : note 1 si la différence entre la moyenne du verrat et la moyenne de référence est négative et supérieure à un demi écart-type de verrat, note 2 si cette différence est comprise entre $-0,5$ et $+0,5$ fois cet écart-type et note 3 si la différence est positive et excède le demi écart-type. L'indice de transformation et le pourcentage de morceaux gras sont affectés du signe - afin que la note 3 corresponde, pour chaque caractère, à un verrat améliorateur.

Une note globale, égale à la somme des 4 notes obtenues ainsi, permet de classer les verrats en 2 catégories : verrats améliorateurs si la note globale est au moins égale à 9 , verrats non améliorateurs si la note est inférieure à 9 .

\section{b) Mєsure de l'intensité de sélection}

Dans une population, l'intensité de sélection (I) peut se mesurer par la différence entre la moyenne des individus sélectionnés et la moyenne générale de la population dont ils sont tirés, différence exprimée en écart-type. En fait, chaque parent sélectionné ne produit pas le même nombre de descendants, ce qui oblige à pondérer la valeur de chaque parent par le nombre de ses descendants.

Pour connaître l'intensité de la sélection pratiquée sur les verrats en fonction des résultats des épreuves de descendance, il faut donc connaitre, pour chacun des verrats mis à l'épreuve, le nombre de ses descendants, mâles et femelles, utilisés dans les élevages de sélection. Les Livres généalogiques porcins établissent, au 1 er janvier de chaque année, la liste des reproducteurs mâles et femelles en service dans les élevages avec mention de leur père. Cette liste permet de connaitre pour chaque verrat le nombre de ses fils et de ses filles utilisés comme reproducteurs à un instant donné. Pour un verrat donné, ce nombre varie dans le temps. Quand le verrat est jeune, ce nombre est faible; il augmente ensuite pour devenir nul après quelques années. Par exemple, si l'on considère tous les verrats soumis à l'épreuve de la descendance en 1960 , on constate que le nombre de leurs descendants en service dans les élevages est maximum au 1 er janvier 1962. Nous avons, par suite, choisi de mesurer le degré d'utilisation d'un verrat par le nombre de ses fils et filles en service dans les élevages de sélection un an après la fin de l'épreuve de descendance.

La mesure de l'intensité de sélection demanderait donc de pondérer la valeur de chaque verrat mis à l'épreuve par le nombre de ses descendants mâles et femelles en service un an plus tard. Une méthode approchée consiste à utiliser les notes définies plus haut au lieu des valeurs réelles de chaque critère. Chaque critère est alors traité comme un caractère à seuil, résultant d'une distribution normale sous-jacente. Connaissant le nombre d'individus dans chaque classe de note $(1,2$ ou 3 ), on peut déduire (FAlconer, 1960) la moyenne $(m)$ et l'écart-type $(s)$ de la distribution sous-jacente. Par ailleurs, on peut calculer la moyenne $\left(m^{\prime}\right)$ des notes des verrats pondérées par le nombre de leurs descendants. On a alors :

$$
\mathrm{I}=\frac{m^{\prime}-m}{s}
$$

Connaissant $I_{m}$ pour les fils et $I_{f}$ pour les filles, l'intensité de sélection est $\left(I_{m}+I_{f}\right) / 2$. Pour un caractère à distribution normale, à une valeur de I correspond un pourcentage $p$ d'individus éliminés tel que

$$
I=\frac{z}{1-p}
$$

$z$ étant l'ordonnée de la courbe normale pour la valeur du caractère correspondant au seuil d'élimination.

Le même principe a été appliqué pour la note globale en considérant seulement 2 classes : améliorateurs (note $\geqslant 9$ ) et non améliorateurs (note $<9$ ). 
Nous allons montrer, à l'aide d'un exemple, la façon de calculer I. Pour le gain moyen quotidien, en race Large White, les 268 verrats mis à l'épreuve se répartissent comme suit : 66 ont la note 1, 110 la note 2 et 92 la note 3. D'après les tables de la distribution normale réduite on déduit que le seuil entre les notes 1 et 2 se trouve à 0,69 écart-type au-dessous de la moyenne de la distribution et le seuil entre 2 et 3 à 0,40 écart-type au-dessus (fig. 1). La largeur de la classe centrale est donc de $(0,69+0,40) s=1,09 s$. Cette largeur correspond à 1 point sur l'échelle de notes adoptée. D'où $s=1 / 1,09=0,91$ point. Si nous attribuons la valeur 1,5 point au premier seuil (et 2,5 points au deuxième), la moyenne de la distribution sous-jacente supposée est :

$$
m=1,5+0,69 s=2,13 \text { points. }
$$

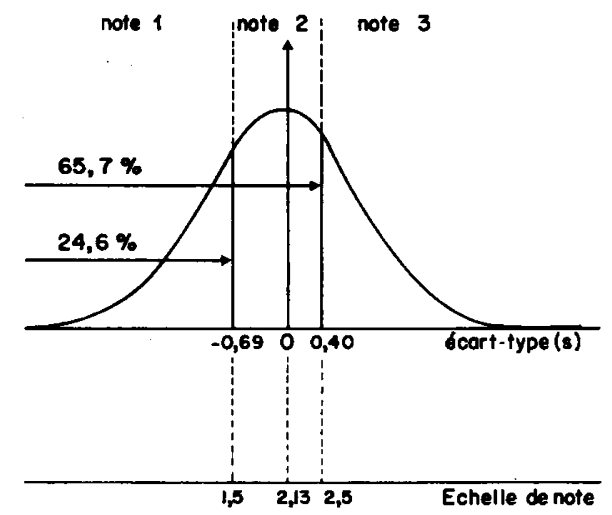

Fig. I. - Exemple d'estimation de la moyenne et de l'écart-type de la distribution des notes de gain moyen quotidien (268 verrats de race Large White).

Par ailleurs, le nombre de fils en service est de 75 pour les 66 verrats ayant la note 1,121 pour les 110 verrats ayant la note 2 et 169 pour les 92 verrats ayant la note 3 . D'où :

$$
\begin{gathered}
m^{\prime}=\frac{1 \times 75+2 \times 121+3 \times 169}{75+121+169}=2,26 \text { points. } \\
I=\frac{m^{\prime}-m}{s}=\frac{2,26-2,13}{0,91}=0,14 \text { écart-type. }
\end{gathered}
$$

\section{RÉSULTATS}

La méthode a d'abord été appliquée aux verrats de race Large White contrôlés de 1956 à I965. Les résultats apparaissent aux tableaux I et 2 . Ce dernier tableau montre que la sélection pratiquée est très faible : $9 \mathrm{p}$. Ioo d'élimination sur le classement global au lieu des 50 p. Ioo théoriquement recommandés aux éleveurs. Une sélection plus forte est appliquée aux critères de carcasse. La sélection effectuée sur les filles des verrats mis à l'épreuve est pratiquement nulle.

En race Landrace, seuls les verrats contrôlés de I958 à I965 ont été considérés (tabl. 3 et 4 ). La sélection effectuée dans cette race est encore plus faible qu'en race Large White, la sélection sur le classement global étant pratiquement nulle. En effet, la sélection va dans le sens désiré en ce qui concerne les critères de carcasse, mais elle est négative pour les critères d'engraissement. 


\section{TABLEAU I}

Nombre de descendants en service par verrat en fonction des résultats de l'épreuve de sa descendance (race Large White)

\begin{tabular}{|c|c|c|c|c|c|c|}
\hline \multirow{2}{*}{ Caractères } & \multirow{2}{*}{ Note } & \multirow{2}{*}{$\begin{array}{c}\text { Nombre } \\
\text { de } \\
\text { verrats }\end{array}$} & \multicolumn{2}{|c|}{$\begin{array}{l}\text { Nombre de fils } \\
\text { en service }\end{array}$} & \multicolumn{2}{|c|}{$\begin{array}{c}\text { Nombre de filles } \\
\text { en service }\end{array}$} \\
\hline & & & Total & Moyen & Total & Moyen \\
\hline Gain moyen quotidien $\ldots \ldots \ldots \ldots$ & $\begin{array}{l}\mathbf{I} \\
2 \\
3\end{array}$ & $\begin{array}{r}66 \\
110 \\
92\end{array}$ & $\begin{array}{r}75 \\
\text { I } 1 \\
169\end{array}$ & $\begin{array}{l}I, I 4 \\
I, I O \\
I, 84\end{array}$ & $\begin{array}{l}528 \\
846 \\
791\end{array}$ & $\begin{array}{l}8,00 \\
7,69 \\
8,60\end{array}$ \\
\hline Indice de transformation $\ldots \ldots \ldots$ & $\begin{array}{l}\mathbf{I} \\
2 \\
3\end{array}$ & $\begin{array}{r}58 \\
124 \\
86\end{array}$ & $\begin{array}{r}40 \\
170 \\
155\end{array}$ & $\begin{array}{r}0,69 \\
\mathbf{1}, 37 \\
\mathbf{1}, 80\end{array}$ & $\begin{array}{r}461 \\
\text { I } 084 \\
620\end{array}$ & $\begin{array}{l}7,95 \\
8,74 \\
7,21\end{array}$ \\
\hline Pourcentage de jambon et longe.. & $\begin{array}{l}I \\
2 \\
3\end{array}$ & $\begin{array}{l}77 \\
96 \\
95\end{array}$ & $\begin{array}{r}50 \\
127 \\
188\end{array}$ & $\begin{array}{l}0,65 \\
1,32 \\
1,98\end{array}$ & $\begin{array}{l}542 \\
735 \\
888\end{array}$ & $\begin{array}{l}7,04 \\
7,66 \\
9,35\end{array}$ \\
\hline Pourcentage de bardière et panne.. & $\begin{array}{l}\mathbf{I} \\
2 \\
3\end{array}$ & $\begin{array}{r}82 \\
102 \\
84\end{array}$ & $\begin{array}{r}59 \\
127 \\
179\end{array}$ & $\begin{array}{l}0,72 \\
1,25 \\
2,13\end{array}$ & $\begin{array}{l}574 \\
796 \\
795\end{array}$ & $\begin{array}{l}7,00 \\
7,80 \\
9,46\end{array}$ \\
\hline Note globale $\ldots \ldots \ldots \ldots \ldots \ldots$ & $\begin{array}{l}\geqslant 9 \\
<9\end{array}$ & $\begin{array}{l}136 \\
132\end{array}$ & $\begin{array}{l}256 \\
109\end{array}$ & $\begin{array}{l}\mathrm{I}, 88 \\
0,83\end{array}$ & $\begin{array}{r}\text { I } 87 \\
978\end{array}$ & $\begin{array}{l}8,72 \\
7,40\end{array}$ \\
\hline Total $\ldots \ldots \ldots \ldots \ldots \ldots$ & & 268 & 365 & 1,36 & 2165 & 8,08 \\
\hline
\end{tabular}

TABLEAU 2

Intensité de sélection par caractère, en écart-type des moyennes de verrats (race Large White)

\begin{tabular}{|c|c|c|c|c|}
\hline Caractère & $\begin{array}{l}\text { Intensité } \\
\text { de sélection } \\
\text { sur les fils }\end{array}$ & $\begin{array}{l}\text { Intensité } \\
\text { de sélection } \\
\text { sur les filles }\end{array}$ & $\begin{array}{l}\text { Intensité } \\
\text { de sélection } \\
\text { moyenne }\end{array}$ & $\begin{array}{c}\text { Pourcentage } \\
\text { d'élimination } \\
\text { équivalent }\end{array}$ \\
\hline $\begin{array}{l}\text { Gain moyen quotidien } \ldots \ldots \ldots \ldots \ldots \\
\text { Indice de transformation } \ldots \ldots \ldots \ldots \\
\text { Pourcentage de jambon et longe } \ldots \ldots \\
\text { Pourcentage de bardière et panne } \ldots \\
\text { Note globale } \ldots \ldots \ldots \ldots \ldots \ldots \ldots\end{array}$ & $\begin{array}{l}0,14 \\
0,24 \\
0,26 \\
0,33 \\
0,29\end{array}$ & $\begin{array}{r}0,00 \\
-0,06 \\
0,06 \\
0,10 \\
0,05\end{array}$ & $\begin{array}{l}0,07 \\
0,09 \\
0,16 \\
0,22 \\
0,17\end{array}$ & $\begin{array}{r}3 \% \\
4 \% \\
8 \% \\
12 \% \\
9 \%\end{array}$ \\
\hline
\end{tabular}


TABLEAU 3

Nombre de descendants en service par verrat en fonction des résultats de l'épreuve de sa descendance (race Landrace)

\begin{tabular}{|c|c|c|c|c|c|c|}
\hline \multirow{2}{*}{ Caractère } & \multirow{2}{*}{ Note } & \multirow{2}{*}{$\begin{array}{c}\text { Nombre } \\
\text { de } \\
\text { verrats }\end{array}$} & \multicolumn{2}{|c|}{$\begin{array}{l}\text { Nombre de fils } \\
\text { en service }\end{array}$} & \multicolumn{2}{|c|}{$\begin{array}{l}\text { Nombre de filles } \\
\text { en service }\end{array}$} \\
\hline & & & Total & Moyen & Total & Moyen \\
\hline Gain moyen quotidien $\ldots \ldots \ldots \ldots$ & $\begin{array}{l}I \\
2 \\
3\end{array}$ & $\begin{array}{l}13 \\
38 \\
20\end{array}$ & $\begin{array}{l}16 \\
41 \\
23\end{array}$ & $\begin{array}{l}1,23 \\
1,08 \\
1,15\end{array}$ & $\begin{array}{r}88 \\
211 \\
108\end{array}$ & $\begin{array}{l}6,77 \\
5,56 \\
5,4^{\circ}\end{array}$ \\
\hline Indice de transformation $\ldots \ldots \ldots$ & $\begin{array}{l}I \\
2 \\
3\end{array}$ & $\begin{array}{l}12 \\
32 \\
27\end{array}$ & $\begin{array}{l}\text { I4 } \\
41 \\
25\end{array}$ & $\begin{array}{l}\mathrm{I}, 17 \\
\mathbf{1 , 2 8} \\
0,93\end{array}$ & $\begin{array}{r}75 \\
193 \\
139\end{array}$ & $\begin{array}{l}6,25 \\
6,03 \\
5,15\end{array}$ \\
\hline Pourcentage de jambon et longe ... & $\begin{array}{l}\mathrm{I} \\
2 \\
3\end{array}$ & $\begin{array}{l}26 \\
18 \\
27\end{array}$ & $\begin{array}{l}28 \\
14 \\
3^{8}\end{array}$ & $\begin{array}{l}1,08 \\
0,78 \\
1,41\end{array}$ & $\begin{array}{r}\text { I I I } \\
89 \\
207\end{array}$ & $\begin{array}{l}4,27 \\
4,94 \\
7,67\end{array}$ \\
\hline Pourcentage de bardière et panne .. & $\begin{array}{l}1 \\
2 \\
3\end{array}$ & $\begin{array}{l}19 \\
28 \\
14\end{array}$ & $\begin{array}{l}17 \\
23 \\
40\end{array}$ & $\begin{array}{l}0,89 \\
0,82 \\
1,67\end{array}$ & $\begin{array}{r}90 \\
141 \\
176\end{array}$ & $\begin{array}{l}4,74 \\
5,04 \\
7,33\end{array}$ \\
\hline Note globale $\ldots \ldots \ldots \ldots$ & $\begin{array}{l}\geqslant 9 \\
<9\end{array}$ & $\begin{array}{l}40 \\
3 \mathrm{I}\end{array}$ & $\begin{array}{l}45 \\
35\end{array}$ & $\begin{array}{l}\mathbf{I}, \mathbf{1} 3 \\
\mathrm{I}, \mathbf{1} 3\end{array}$ & $\begin{array}{l}244 \\
163\end{array}$ & $\begin{array}{l}6,10 \\
5,26\end{array}$ \\
\hline Total. . & & 71 & 80 & 1,13 & 407 & 5,75 \\
\hline
\end{tabular}

TABLEAU 4

Intensité de sélection par caractère, en écart-type des moyennes de verrats (race Landrace)

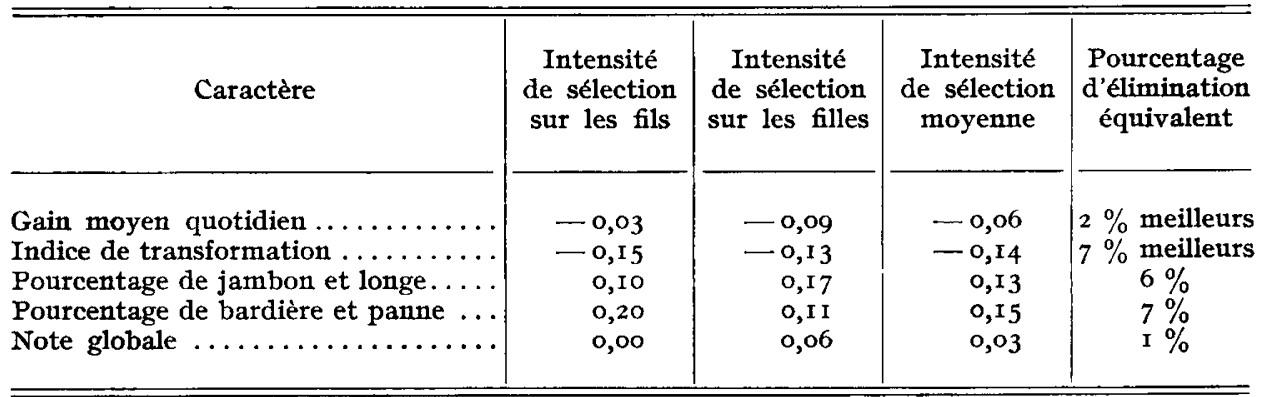




\section{DISCUSSION ET CONCLUSIONS}

Les estimations d'intensité de sélection qui viennent d'être présentées ne tiennent pas compte de l'âge des verrats au moment de leur mise à l'épreuve. Or, il est bien évident que les verrats très jeunes ou très vieux ont, du fait même de leur âge, moins de descendants en service un an après l'épreuve que les verrats d'âge moyen. Il serait possible théoriquement d'appliquer des facteurs de correction en fonction de l'âge, estimés sur des verrats non soumis à l'épreuve de descendance. Mais si l'on suppose que la répartition par âge des verrats mis à l'épreuve est indépendante des résultats de cette épreuve, on peut admettre que les estimations ci-dessus ne sont entachées d'aucune erreur systématique.

Cette étude ne permet pas de dire dans quelle mesure les caractères considérés ont pu être améliorés génétiquement au cours des dix années considérées. S'il y a eu amélioration, elle n'a pas résulté de l'utilisation des épreuves de descendance. On pourrait, dans ce cas, invoquer soit une sélection sur la conformation, soit l'importation de géniteurs étrangers. La première méthode est présumée peul efficace et d'autre part le nombre des reproducteurs importés est faịble, ce qui conduit à supposer que le progrès génétique a été faible, sinon nul, au cours de la période considérée. Une étude visant à l'évaluer exactement est en cours. D'autre part, cette étude démontre que l'attention portée par les éleveurs aux résultats de carcasse 1 'a souvent été au détriment de la vitesse de croissance et de l'indice de consommation, ce qui a affaibli la sélection pratiquée sur la note globale. Ceci indique qu'il est souhaitable de synthétiser les résultats des épreuves de la descendance sous forme d'une note unique (par exemple un index) qui doit, seule, intervenir dans la sélection.

Les sélectionneurs français ont donc, dans le passé, peu tenu compte des résultats des épreuves de descendance dans le choix de leurs reproducteurs. Une étude effectuée par Sмrтr (I965) aboutit, par des méthodes différentes, à des estimations d'intensité de sélection très voisines. Il semble que, dans l'espèce porcine, cette méthode de sélection, au demeurant peu efficace sur le plan théorique (DICKERSON et HAZEL, I944), soit difficile à utiliser correctement dans la pratique. L'examen détaillé des résultats qui ont servi à cette étude montre cependant que l'intensité de sélection s'est légèrement accrue ces dernières années.

Il n'est peut-être pas inutile de rappeler, en conclusion, la nécessité de s'assurer de la bonne utilisation des techniques de sélection proposées, surtout quand elles supposent des moyens financiers importants, en soumettant les utilisateurs à une réglementation très stricte dans le choix de leurs reproducteurs.

Reçu pour fublication en octobre 1968.

\section{REMERCIEMENTS}

Nous tenons à remercier M. J. Owen, Directeur des Livres généalogiques porcins, qui a bien voulu mettre ses archives à notre disposition pour réaliser cette étude. 


\section{SUMMARY}

NOTE ON THE USE OF PROGENY TEST RESULTS BY PIG BREEDERS

IN FRANCE BETWEEN I956 AND I965.

A simple method is proposed for measuring objectively the selection intensity practised in light of the results of the progeny tests. This method consists of weighting the score given to each boar at the conclusion of the test by the number of his sons and daughters actually used as breeding stock in seedstock herds one year after the test.

It appears that in France from 1956 to 1965 the results of the progeny tests have had a much more direct influence on the choice of males than on the choice of females, and that selection on carcass score has been more intense than on fattening score. However, selection on the overall score is weak, since in the Large White breed the selection practiced was equivalent to culling the lowest $9 \%$ of the boars tested, while in the Landrace selection was essentially nil.

\section{RÉFÉRENCES BIBLIOGRAPHIQUES}

Dickerson G. E., HAzEL I. N., r944. Effectiveness of selection on progeny performance as a supplement to earlier culling in livestock. J. agric. Res., 69, 459-476.

FALCONER D. S., I960 Introduction to quantitative genetics. Oliver et Boyd, Irondon.

Sмrтн C., 1965. Results of pig progeny testing in Great Britain. Anim. Prod., 7, 133-140. 